Provided by the author(s) and University of Galway in accordance with publisher policies. Please cite the published version when available.

\begin{tabular}{|c|l|}
\hline Title & $\begin{array}{l}\text { Electrophysiological correlates of flicker-induced color } \\
\text { hallucinations }\end{array}$ \\
\hline Author(s) & Elliott, Mark \\
\hline $\begin{array}{c}\text { Publication } \\
\text { Date }\end{array}$ & 2009-03 \\
\hline $\begin{array}{c}\text { Publication } \\
\text { Information }\end{array}$ & $\begin{array}{l}\text { Becker, C., Gramann, K., Müller, H. J., \& Elliott, M. A. } \\
\text { hallucinations. Consciousness and Cognition, 18(1), 266-276. }\end{array}$ \\
\hline $\begin{array}{c}\text { Publisher } \\
\text { halloger }\end{array}$ & Elsevier \\
\hline $\begin{array}{c}\text { Link to } \\
\text { publisher's } \\
\text { version }\end{array}$ & http://dx.doi.org/10.1016/j.concog.2008.05.001 \\
\hline $\begin{array}{c}\text { Item record } \\
\text { http://hdl.handle.net/10379/1528 }\end{array}$ \\
\hline
\end{tabular}

Downloaded 2023-04-26T12:40:43Z

Some rights reserved. For more information, please see the item record link above.

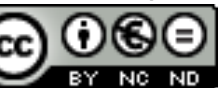




\title{
Electrophysiological correlates of flicker-induced color hallucinations
}

\author{
Cordula Becker ${ }^{\mathrm{a}, \mathrm{c}, *}$, Klaus Gramann ${ }^{\mathrm{b}, \mathrm{c}}$, Hermann J. Müller ${ }^{\mathrm{c}}$, Mark A. Elliott ${ }^{\mathrm{c}, \mathrm{d}}$ \\ a Department of Biopsychology, Faculty of Psychology, Ruhr-University Bochum, D-44780 Bochum, Germany \\ ${ }^{\mathrm{b}}$ Swartz Center for Computational Neuroscience, University of California, San Diego, USA \\ ${ }^{\mathrm{c}}$ Abteilung für Allgemeine und Experimentelle Psychologie, Department Psychologie, Ludwig-Maximilians-Universität München, Germany \\ d School of Psychology, National University of Ireland Galway, Ireland
}

\section{A R T I C L E I N F O}

\section{Article history:}

Received 4 January 2008

Available online $\mathrm{xxxx}$

\section{Keywords:}

EEG

Alpha band

Gamma band

Hallucinatory color

Flicker

\begin{abstract}
A B S T R A C T
In a recent study, Becker and Elliott [Becker, C., \& Elliott, M. A. (2006). Flicker induced color and form: Interdependencies and relation to stimulation frequency and phase. Consciousness E Cognition, 15(1), 175-196] described the appearance of subjective experiences of color and form induced by stimulation with intermittent light. While there have been electroencephalographic studies of similar hallucinatory forms, brain activity accompanying the appearance of hallucinatory colors was never measured. Using a priming procedure where observers were required to indicate the presence of one of eight target colors we compared electrophysiological correlates of hallucinatory color with brain states associated with other visual phenomena. Different target colors were accompanied by different patterns of EEG activation. However, in general, we found that the appearance of hallucinatory colors is preceded by a power decrease in the lower alpha band alongside an increase in gamma band frequencies. We argue that decreasing activity in the lower alpha band acts as a gating mechanism, inducing a switch in perception between different colors. The increasing gamma activation may correlate with the formation of a coherent conscious percept.
\end{abstract}

(c) 2008 Elsevier Inc. All rights reserved.

\section{Introduction}

Conscious visual experience relies on the interaction of our nervous system with light reflected from spatial structures in the environment. It is often assumed that the visual experience is directly related to spatial or spectral characteristics of the external stimulus, so that, for example, spatial properties of the external stimulus are directly mapped onto spatial properties of the internal experience. However and as recently shown by Becker and Elliott (2006), hallucinatory visual experiences are a class of phenomenal experience which cannot necessarily be mapped directly onto the spatial and spectral properties of a stimulus. Becker and Elliott showed that experience of hallucinatory colors and forms can be reliably induced by the uniform stimulation of the visual field with flickering light. These hallucinatory phenomena were highly complex in nature, and seemed, on the basis of participants' reports to be relatively uniformly distributed across the whole visual field. Analysis of the number of reported hallucinatory experiences over a range of stimulation frequencies revealed that the report distribution for each class of hallucinatory experiences was restricted to particular subranges. In addition, peak frequencies, i.e. those most effective in inducing hallucinatory phenomena were found to vary according to the type and set (form or color) within which a given hallucinatory phenomenon could be naturally classified (Becker \& Elliott, 2006). Taken as a whole, forms were reported across the $8-40 \mathrm{~Hz}$ range (median frequency: $25 \mathrm{~Hz}$ ) while colors were reported across the range 5-56 $\mathrm{Hz}$ (median frequency: $30 \mathrm{~Hz}$ ). Of particular interest was the relationship between hallucinatory form and color, with forms often defined by either having colored contours or appearing entirely filled with color.

\footnotetext{
* Corresponding author. Address: Department of Biopsychology, Faculty of Psychology, Ruhr-University Bochum, D-44780 Bochum, Germany.

E-mail address: cordula.becker@rub.de (C. Becker).
} 
While there have been some electroencephalographic (EEG) studies of hallucinatory form (Shevelev, Kamenkovich, \& Sharaev, 1996; Shevelev et al., 2000), the electrophysiological correlates of hallucinatory color have not yet been investigated. In most models, experience of hallucinatory color is attributed to early stages of visual processing (e.g. Courtney \& Buchsbaum, 1991; Grunfeld \& Spitzer, 1995). However, for conscious experience of the hallucination processing that extends to later, extra-sensory cortical areas would be necessary: This is evidenced by Billock and Tsou (2007) who showed that (flicker-induced) hallucinatory-pattern formation is strongly influenced by the presentation of adjacent geometrical forms, thereby indicating that similar cortical processes cooperate to bring about both normal vision, and hallucinatory perception.

We aimed to investigate the EEG recorded from the scalp for evidence of the cortical processes correlated with the emergence of hallucinatory color and to compare these findings against electrophysiological processes described for a variety of well-studied visual phenomena. The scalp recorded EEG is an index of the synchronized activation of neuronal assemblies in the brain and the extent of synchronization at particular frequencies can be measured in the EEG power spectra. Here, increased power accompanies stronger synchronization while desynchronization is marked by a decrease in power (Klimesch, 1999). By convention, EEG activity is divided into several frequency bands with different authors delimiting slightly different bands with slightly different functional characteristics. However, Klimesch $(1997,1999)$ has recommended determining each band individually for each subject because of natural variation in the ranges over which the EEG bands vary. This recommendation carries with it the implication that using standardized bands for the EEG analysis would lead to noisy signals and a loss of information on their functional characteristics due to an incorrect assignment of the frequency of EEG activity to a particular bandwidth. Individually derived bands allow detection of band-specific activations even with individually differing EEG-frequency distributions.

Different frequency bands have been ascribed different cognitive or perceptual functions (see, e.g. the review by Başar, Başar-Eroglu, Karakaş, \& Schürmann, 1999) with two frequency bands attributed a particular role in perceptual processing. These are the alpha and gamma EEG bands representing synchronized cortical activity between approximately $8-12 \mathrm{~Hz}$ and $30-70 \mathrm{~Hz}$, respectively. Alpha activity accompanies a relaxed awake state with closed eyes (Niedermeyer, 1999) and sensory stimulation usually brings about a decrease in alpha power and is thus claimed to represent a state of cognitive activation (Klimesch, 1999). It has been shown that the alpha band $(8-13 \mathrm{~Hz})$ can be divided into two bands, the lower and upper alpha bands which cover the ranges $8-10 \mathrm{~Hz}$ and $11-13 \mathrm{~Hz}$, respectively. These frequency bands present themselves as statistically independent (Mecklinger, Kramer, \& Strayer, 1992) and are claimed to have precise functional non-equivalence (Klimesch, 1997).

More generally the alpha band is associated with visual detection and discrimination performance including the perception of multistable figures; Ergenoglu et al. (2004) have shown that the relative power of the EEG alpha band was low in trials in which a near-threshold stimulus was detected as compared to trials in which the stimulus could not be detected by the participants. The finding that good perceptual performance is related to low alpha power has also been shown by Hanslmayr et al. (2005). A variety of studies have shown that decreasing alpha power precedes the transitions between alternative perceptual representations of a multistable visual stimulus (Isoglu-Alkaç et al., 2000; Müller, Federspiel, Fallgatter, \& Strik, 1999; Müller et al., 2005), findings confirmed in using magnetoencephalographic (MEG) measurements by Strüber and Herrmann (2002). Evidence for the potential of alpha activity for binding separate neural processes has been provided by von Stein and Sarnthein (2000, also von Stein, Chiang, \& König, 2000) who have reported long-range fronto-parietal interactions in the theta and alpha frequency band during working memory retention and mental imagery. Gamma band synchronization during visual processing appeared to be largely confined to localized interactions and is more generally associated with the perceptual binding of stimulus features and memory processing (Başar-Eroglu, Strüber, Schürmann, Stadler, \& Başar, 1996b). One set of findings by Tallon, Bertrand, Bouchet, \& Pernier, 1995, also Tallon-Baudry \& Bertrand, 1999) support this hypothesis, in revealing an increase in $30-\mathrm{Hz}$ power following the presentation of relatively good visual groupings, but not when non-good groupings were presented. Gamma activity is often considered in relation to attentional demands. This follows the popular idea that coherent visual perception is a function of attentional deployment and finds some support in recordings of a larger EEG response at around $40 \mathrm{~Hz}$ when attentional resources are engaged (Engel, Fries, \& Singer, 2001; Herrmann \& Mecklinger, 2001; Tiitinen et al., 1993). However, increases in gamma coherence also accompany states of high expectancy and feature attention, even before any stimulus is presented (see Engel et al., 2001, for a review). Gamma activity may be distributed rather widespread over the brain, and may be thus assumed to be a generalizable functional bandwidth with, perhaps different functional correlates in perception depending on its location and generators.

In summary, a desynchronization of alpha combined with the synchronization of gamma activity may be a prerequisite for conscious perception, including the registration, or availability in phenomenal awareness of internal representations generated by external visual stimuli. Here and in relation to hallucinatory flicker-induced experiences the question arises as to whether changes in alpha and gamma activity are involved during experience of flicker-induced hallucinatory colors. The aim of this study is to investigate EEG responses during and immediately preceding experience of hallucinatory colors with a view to determining the particular contributions of EEG alpha and gamma activity to the experience of hallucinatory color.

\section{Materials and methods}

\subsection{Participants}

Three healthy practiced volunteers (all female, mean age 26 years) participated in the experiment. All participants had prior experience with the experimental paradigm and were chosen on the basis of being reliable reporters of hallucinatory 
color experiences under flicker conditions. All had normal or corrected to normal vision. Participant CB and KM were lefthanded, participant KO was right-handed. Participants KM and KO were paid for their participation at a rate of 8 Euro per hour.

\subsection{Stimulation}

Flicker was presented using a specially constructed box with four diodes placed in the upper and lower left and right of a single viewing aperture. The diodes were screened from view and projected onto a uniformly white screen (of $10 \times 20 \mathrm{~cm}$ ) mounted within the box some $12 \mathrm{~cm}$ from the viewing aperture. Aside from the viewing aperture, which was molded to fit a standard facial physiognomy, there were no sources of external light in the box. During stimulation, the screen was illuminated by rapid and intermittent square-wave light pulses of $13 \mathrm{~cd} / \mathrm{m}^{2}$ emitted simultaneously from each of the four diodes. Using the viewing aperture, participants viewed the projection of these pulses of light on the white screen, which for all presentation frequencies was experienced as a ganzfeld of uniform (that is spatially homogeneous) flicker. Verbal instructions via headphones, stimulus generation, and response collection were all ensured by a PC running under MSDOS and generated in the $C$ programming language. The precise temporal delivery of light pulses was achieved using a PCI technology timer card (CIO-CTR05 with CTS9513 chip capable of temporal resolutions of up to $5 \mathrm{kHz}$ ) mounted in a conventional IBM compatible PC running under MSDOS and connected to the four LEDs (light emitting diodes; Type WU-2-310SWC-UR, luminous intensity $900 \mathrm{mcd}, 3 \mathrm{~mm}$ diameter, emission color white, CIE 1931 Standard: $x=0.29, y=0.30$, Conrad Electronic GmbH, 92240 Hirschau, Germany, Order No. 153881-62).

All experiments were conducted in accordance with the code of ethics of the World Medical Association (the Declaration of Helsinki, 2000), under the guidelines of the American Psychological Association, and following the approval of the Departmental Research Ethics Committee. All participants gave written informed consent to their participation in the study and had no prior history of neurological or neuroleptic disorders.

\subsection{Design and procedure}

The study used a paradigm in which participants were asked to press a response key with the left index finger as quickly as possible on first experiencing a particular target color which was announced before the beginning of the trial. This paradigm was employed with the aim to directly link EEG-frequency changes to the occurrence and report of a specific hallucinatory color. Previous experiments (Becker \& Elliott, 2006) have shown that the distribution of color experiences over stimulation frequency are comparable for a free-report and a primed response-time paradigm with a slight overall increase in reports for the primed response-time design.

The target color was announced to participants in their native language which was German in the form of a verbal instruction (red [rot], blue [blau], green [grün], yellow [gelb], purple [violett or lila], white [weiss], black [schwarz], gray [grau], German names given in square brackets). The target was announced via headphones and immediately prior to trial onset. Target colors were those colors reported most reliably in the free-report study described in Becker and Elliott (2006, Experiment 1). These colors have also been tested successfully in a primed response-time paradigm similar to the one employed in the present study (Becker \& Elliott, 2006, Experiment 2) and distributions over flicker frequency were found to be very similar. Participants were reminded before each session that a response should only be given to the unequivocal experience of the target color. In the case of a response the flicker presentation terminated with the button press. When the participant did not experience the target color the trial was allowed to time out and no response-time was recorded. After completion of a trial (with or without response) the participants were asked to name all other colors which they experienced during the flicker. These experiences were then transcribed by the experimenter who was not aware of the target color or of the flicker frequency for the trial concerned.

Each trial consisted of a 20-s epoch of no stimulation serving as a baseline for the EEG recording, followed by an epoch of no more than $20 \mathrm{~s}$ of flicker. The verbal instruction was always given before the baseline, ensuring that the memory load of remembering the target color was similar during baseline and epochs of flicker. The beginning of the next trial was always initiated by the experimenter. Frequencies between 13 and $20 \mathrm{~Hz}$ which were shown to be the most effective in inducing hallucinatory color were tested (Becker \& Elliott, 2006). The frequency of flicker was maintained as trial-wise constant but was varied between trials. Both the presentation order of the eight different flicker frequencies and the eight different target hallucinatory colors were varied pseudo-randomly over trials on a participant by participant and session by session basis. Every frequency $\times$ color combination was tested twice in the course of the experiment resulting in 128 trials over the entire experiment for each participant.

\subsection{EEG recordings}

The EEG was recorded from $64 \mathrm{Ag}-\mathrm{AgCl}$ electrodes placed according to the extended $10-20$ system. EEG was sampled at a rate of $500 \mathrm{~Hz}$. The electrodes were mounted in an elastic cap (Easy Cap, Munich, Germany) and were referenced to $\mathrm{Cz}$ with electrode impedance kept below $5 \mathrm{k} \Omega$. The EOG was registered with standard electrodes F9 and F10 at the outer canthi of each eye serving as horizontal EOG. Electrodes Fp1 and one additional electrode below the left eye served as vertical EOG. EEG activity was amplified using BrainAmpMR amplifiers (BrainProducts, Munich, Germany) and were lowpass filtered 
at $1 \mathrm{~Hz}$ and highpass filtered at $100 \mathrm{~Hz}$. The following 18 electrodes were included in the later analyses: F5, Fz, F6, T7, C5, C6, T8, P5, Pz, P6, PO7, PO3, Poz, PO4, PO8, O1, Oz, and O2.

\subsection{Methods of analysis}

To account for the individual differences in electrophysiological responses, analyses were conducted separately for each participant and then compared for inter-individually consistent responses.

The flicker frequencies presented in this study are associated with a very high probability of color hallucinations (for the colors studied), however the results of Becker and Elliott (2006) suggest that no differences in the distributions of different color hallucinations over flicker frequency were to be expected and, consistent with this expectation no significant differences were found [Friedman test, $\chi^{2}(7)=6.661$; the median frequencies being of $15 \mathrm{~Hz}$ (gray), $16 \mathrm{~Hz}$ (white, black, green, purple) and $17 \mathrm{~Hz}$ (red, blue, yellow)]. The data was collapsed over the different stimulation frequencies for subsequent analyses.

Target colors were reported on 75\% (participant $\mathrm{CB}$ ), 76.6\% (KM) and 53.9\% (KO) of the 128 trials. Additional, non-target colors freely reported following $90.5 \%$ of these trials with participants reporting on average 1.13 (CB), 1.93 (KM) or 1.55 (KO) additional colors.

On $97.5 \%$ of all trials where the target color was not reported, other colors than the target color were perceived and reported following the trial. The mean number of colors per trial reported in trials where no target color was reported, was 2.16 (CB), $2.03(\mathrm{KM})$ and $2.34(\mathrm{KO})$. On this basis and because the pattern of emergence of particular colors over stimulation frequency can only be guessed on the strength of previous data (i.e. Becker \& Elliott, 2006) the no-report trials were not considered suitable as control data and were excluded from further analysis.

Three stimulation epochs were defined within each trial: (i) a baseline epoch which lasted $2000 \mathrm{~ms}$ starting $10 \mathrm{~s}$ after trial onset and during which there was no stimulation, (ii) a baseline epoch of flicker during which no response was given. (Reports of hallucinatory color were given during this epoch on only $7 \%$ of the trials and so this epoch is characterized by a low probability of hallucinatory color being experienced: these trials were removed for the purposes of baseline calculation). To avoid artefacts induced by the flicker onset, this epoch started $500 \mathrm{~ms}$ after flicker onset and lasted for $2000 \mathrm{~ms}$. (iii) The flicker epoch immediately preceding the response to a hallucinatory color with a duration of $2000 \mathrm{~ms}$.

A fast Fourier transformation (FFT) using a Hanning window (10\%) was calculated on these three epochs for each trial separately. The frequency resolution of the FFT was set to $0.5 \mathrm{~Hz}$, and applied to the normalized data for the frequency range 1-100 Hz. The FFT was calculated on time windows of $512 \mathrm{~ms}$ shifted along the $2000 \mathrm{~ms}$ epochs in $100 \mathrm{~ms}$ steps. Following the FFT the mean power values for all trials were calculated separately for each participant, each color, each EEG-frequency bin (of $0.5 \mathrm{~Hz}$ ) and each of the 20 periods of the three stimulation epochs.

The individual alpha frequency (IAF) ranges were determined by the method developed and described by Klimesch (1997). For the baseline epoch, power spectra were calculated for each participant and each of the 18 electrodes, collapsed over all trials. By visual inspection, the beginning of the ascent and the end of the descent of the alpha peak were derived for each participant and electrode. The individual alpha bandwidths were calculated as the mean of the thus determined IAF ranges over all electrodes. The lower and upper limits of the individual alpha bandwidths are $7-12.5 \mathrm{~Hz}$ (participant $\mathrm{CB}$ ), 8-13.5 Hz (KM) and 7-14 Hz (KO).

The IAF range was divided into two equal parts to form the lower alpha frequency range and the upper alpha frequency range (i.e. 7-9.5 and 10-12.5 Hz, respectively, for participant $\mathrm{CB}, 8-10.5$ and $11-13.5 \mathrm{~Hz}$ for $\mathrm{KM}, 7-10.5$ and $11-14 \mathrm{~Hz}$ for KO). Based on the literature (e.g. Başar et al., 1999; Klimesch, 1999), the gamma EEG-frequency range was defined on the basis of the IAF ranges as consisting of a $30 \mathrm{~Hz}$ range $10 \mathrm{~Hz}$ above the upper alpha frequency range (i.e. $23.5-53.5 \mathrm{~Hz}$ for participant $\mathrm{CB}, 24.5-54.5 \mathrm{~Hz}$ for $\mathrm{KM}, 25-55 \mathrm{~Hz}$ for $\mathrm{KO}$ ).

As the study aimed to investigate electrophysiological correlates of hallucinatory color, it was necessary to eliminate extraneous variance in the EEG arising from procedural aspects of the experiment (e.g. the rehearsal of prespecified target colors) and the first order effects of flicker on visual and other brain systems. As described above, activity during the baseline epoch (i.e. no stimulation) was used to determine the individual frequency bands based on the IAF (Klimesch, 1997, 1999). The flicker baseline epoch may account for individual activation differences and activations due to memory processes during the holding of the target color in memory and is assumed to generate activity which is specific to stimulation with flickering light that is not (yet) accompanied by hallucinatory experience. In contrast, activation during the flicker epoch shortly before a response is given is thought to contain additional activity specific for the experience of hallucinatory color. The power values for each participant, each color, each frequency bin and each of the 20 periods were corrected by subtraction of the flicker baseline activation from the activity in the flicker epoch preceding a response.

The corrected power values were then subject to regression analyses against time. These analyses were done separately for each participant, each color, each of the 18 electrodes and each of the 3 analyzed frequency bands (lower alpha, upper alpha, gamma). The aim of these analyses was to determine changes in the power of different EEG-frequency bands over the time interval of $2000 \mathrm{~ms}$ preceding the hallucinatory experience. The EEG power values were assumed to increase or decrease significantly when the slope of the regression had a positive or negative sign (for increases or decreases, respectively) and was significant with $\alpha<.05$ (see Fig. 1 for an example). 


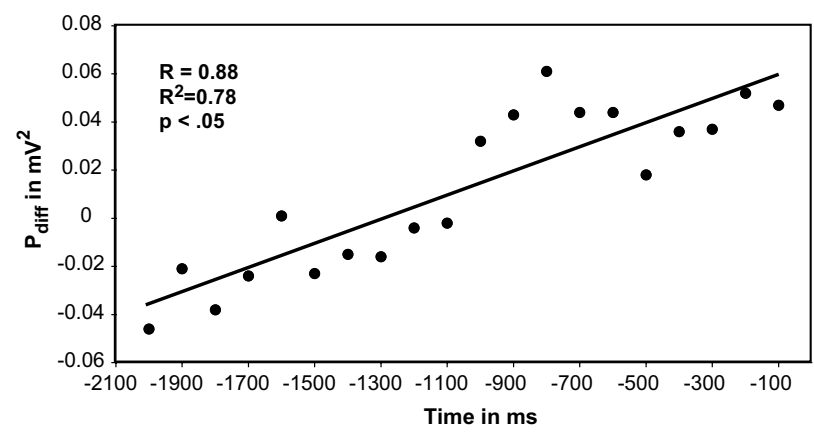

Fig. 1. Regression of the corrected EEG power over time for participant KM in the gamma frequency band at electrode O2. The regression analysis was conducted over $2000 \mathrm{~ms}$ of the flicker epoch preceding the response (and the corresponding periods for the baseline flicker epoch). The response occurred at time zero. The correlation coefficient, the coefficient of determination and the significance of the regression are given in the upper left of the figure.

\section{Results}

\subsection{Electrophysiological correlates of different hallucinatory colors}

\subsubsection{Individual analyses}

The probabilities of report for all target colors and all participants are given in Table 1 . A contingency $\chi^{2}$-test revealed significant differences between participants in the probabilities of report for the different target colors. CB was more likely to report black and blue compared to the expected average between observers, but less likely to report yellow. KM showed fewer reports of red than expected, while KO was less likely to report black and blue, but had a higher likelihood of reporting red and yellow than expected. Complex patterns of power changes for the different colors, frequency bands and electrodes were observed for each participant and are detailed in Tables 2-4.

\subsubsection{Between-participants comparisons}

The aim of the between-participants comparisons was to derive indices of inter-individual reliability as regards changes in EEG power for each of the frequency bands of interest. For each color, at each electrode and in each frequency band, a change in EEG power was assumed to be reliable if it was present and had the same sign in all three participants. The results of the analysis are displayed in Fig. 2. The report of white, black, blue and yellow was accompanied by a right parieto-occipital increase in gamma synchronization, while the report of black and purple was marked by a left parieto-occipital increase in gamma power. Gamma power decreased fronto-centrally for the perception of black. A decrease of power in the upper alpha range was observed for black and yellow at parieto-central electrodes. The report of gray and red was marked by a left temporal decrease of lower alpha activation, while red was also accompanied by a lower alpha desynchronization at right parieto-occipital electrodes. Black and blue were similarly marked by a right parieto-occipital decrease of lower alpha activation, with blue showing a rather widespread lower alpha desynchronization over the entire parieto-occipital and right temporal cortex. A right temporal decrease of lower alpha synchronization was observed for purple.

\subsection{Electrophysiological correlates of hallucinatory color independent of color category}

A second analysis was conducted without differentiation between the different colors: responses to different colors were collapsed and treated in terms of a singular variable 'color'. As such, this analysis targets neural correlates of the experience of hallucinatory color independent of the precise color category. This analysis, while violating clear phenomenological discrepancies between the experience of different colors is nonetheless consistent with the distribution of reports which did not differ in frequency over flicker frequency. As such, this analysis examines for a generalizable neural correlate of hallucinatory color experience. The methods of analysis were equivalent to the analyses outlined above, with the difference that the mean power values were calculated separately for each participant, each EEG-frequency bin (of $0.5 \mathrm{~Hz}$ ) and each of the 20 periods of the three stimulation epochs, but were collapsed over all target colors.

Table 1

Probabilities of report in \% for all target colors and all participants

\begin{tabular}{|c|c|c|c|c|c|c|c|c|}
\hline & White & Black & Gray & Red & Green & Blue & Yellow & Purple \\
\hline $\mathrm{CB}$ & 63 & 94 & 56 & 63 & 100 & 100 & 50 & 75 \\
\hline KM & 88 & 63 & 69 & 50 & 81 & 75 & 100 & 88 \\
\hline KO & 44 & 0 & 63 & 94 & 75 & 25 & 94 & 38 \\
\hline
\end{tabular}

The total number of trials for each target color and each participant was 16. 
Table 2

Electrodes showing significant increases $(+)$ and decreases $(-)$ of corrected EEG power for the different frequency bands and colors in participant $\mathrm{CB}$

\begin{tabular}{|c|c|c|c|c|c|c|c|c|c|c|c|c|c|c|c|c|c|c|}
\hline & F5 & $\mathrm{T} 7$ & C5 & P5 & PO7 & PO3 & 01 & $\mathrm{Fz}$ & $\mathrm{Pz}$ & $\mathrm{POz}$ & $\mathrm{Oz}$ & $\mathrm{O} 2$ & PO4 & PO8 & P6 & C6 & T8 & F6 \\
\hline \multicolumn{19}{|c|}{ Lower alpha } \\
\hline White & - & - & - & - & - & - & - & & - & - & - & & & & & - & - & - \\
\hline Black & - & - & - & - & - & - & - & & - & - & & - & - & - & - & - & - & - \\
\hline Gray & - & - & - & & & & 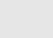 & & & & & & & & & & & \\
\hline Red & & - & - & & & - & & & - & - & - & - & - & - & - & & & \\
\hline Green & & & & - & - & & - & & & & & & & & & & - & \\
\hline Blue & - & - & - & - & - & - & - & + & - & - & - & - & - & - & - & - & - & - \\
\hline Yellow & & & & + & + & + & + & & & + & + & + & + & & & & & \\
\hline Purple & - & - & - & - & - & - & - & & - & - & - & - & - & - & - & - & - & - \\
\hline \multicolumn{19}{|c|}{ Upper alpha } \\
\hline White & & - & - & - & - & - & - & + & - & - & - & - & - & - & - & - & - & 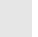 \\
\hline Black & & + & & & & 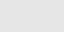 & & & - & & & & & + & + & & & + \\
\hline Gray & + & + & + & + & + & + & + & & + & + & + & + & + & + & + & + & + & \\
\hline Red & - & - & & - & - & - & - & & - & - & - & - & - & - & - & - & - & - \\
\hline Green & & & & & & & & & & - & & & - & & & & & \\
\hline Blue & & & & & - & & & + & - & & & & - & & & & & \\
\hline Yellow & & + & & & & - & & & - & - & & & - & & & & & \\
\hline Purple & - & - & - & & - & & - & + & & - & - & - & & & & - & - & \\
\hline \multicolumn{19}{|l|}{ Gamma } \\
\hline White & & & & & & & & - & & & & & & + & & + & & \\
\hline Black & & & & + & + & + & + & - & + & & & & + & + & + & + & + & + \\
\hline Gray & & + & & & & - & & & - & - & & & - & & & & & - \\
\hline Red & + & + & + & + & + & + & + & - & + & + & + & + & + & + & + & + & + & + \\
\hline Green & + & & & & & & - & - & & & & & & + & & & & \\
\hline Blue & + & + & + & + & & & & - & & & & & & + & + & + & + & + \\
\hline Yellow & + & + & + & & & + & + & 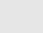 & + & + & + & + & + & + & + & + & + & \\
\hline Purple & + & + & + & + & + & + & + & - & & & & + & + & + & + & + & + & + \\
\hline
\end{tabular}

\subsubsection{Individual analyses}

The target colors were reported in 75\%, 76.6\% and 53.9\% of all trials by participant CB, KM and KO, respectively. $22.9 \%$, $4.1 \%$ and $2.9 \%$ of these responses were not accompanied by other color reports. The mean number of about two non-target colors (CB 2.16, KM 2.03 and KO 2.34) being reported in trials where no target color was reported indicates a higher likelihood for report of the target color than any non-target color (the probability of report for a non-target color being roughly 2 out of 7, i.e. 30\%). However, this increased likelihood of report does not necessarily imply a higher likelihood for target colors to be perceived. The hallucinatory experiences being highly complex and dynamic in nature (Becker \& Elliott, 2006), it is conceivable that while any color will be experienced in about 54-77\% of all trials, only about half of these experiences will be reported using a free-report paradigm.

Regression analysis of EEG power over time revealed patterns of significant power increases and decrease for the different frequency bands of the EEG as illustrated in Fig. 3 (dark gray values indicate a decrease in EEG band power while white values indicate an increase).

\subsubsection{Comparative analyses between participants}

At each electrode and in each frequency band, a change in EEG power was assumed to be reliable if it was present and had the same sign in all three participants. No inter-individually reliable changes in power over time were found for EEG responses in the upper alpha band. In the lower alpha band a generalizable pattern of EEG activity immediately preceding the response was present, characterized by a bilateral decrease of activation under frontal, central, temporal and occipital electrodes, and a right-hemispheric decrease under parietal, and parieto-occipital electrodes (Fig. 4). In addition, increasing gamma power was observed under central, temporal, parieto-occipital, and occipital electrodes. Slight differences in activation were revealed between the two hemispheres in the lower alpha band with a more widespread decrease of power over the right hemisphere. In contrast, changes of activation in the gamma band were not hemisphere-specific, but distributed widely over the cortex. ${ }^{1}$

\footnotetext{
${ }^{1}$ An alternative analysis taking into account the dominant hand of the observer yields slightly different results. While CB and KM were left-handed, KO was right-handed. Therefore, responses on the left hemisphere in CB and KM were matched to right hemispheric responses in KO with the analysis otherwise being the same as before. Consistent activity patterns found in all three participants are now defined in terms of ipsi- or contralaterality to the dominant hand of the observer. No inter-individually reliable changes of activity were found in the upper alpha frequency band. In the lower alpha band EEG activity immediately preceding the response was characterized by a bilateral decrease of activation under frontal (F5, F6), central (C5, C6), temporal (T7, T8) and occipital electrodes $(01,02)$, and a decrease under parietal, and parieto-occipital electrodes ipsilateral to the dominant hand (P6 and PO8, or P5 and PO7). Increasing gamma power was observed under parieto-central electrodes (Pz), bilateral under parieto-occipital (PO7, PO8) and occipital electrodes (01, 02), and contralateral to the dominant hand under temporal and central electrodes (C6 and T8, or C5 and T7).
} 
Table 3

Electrodes showing significant increases $(+)$ and decreases $(-)$ of corrected EEG power for the different frequency bands and colors in participant KM

\begin{tabular}{|c|c|c|c|c|c|c|c|c|c|c|c|c|c|c|c|c|c|c|}
\hline & F5 & $\mathrm{T} 7$ & C5 & P5 & PO7 & $\mathrm{PO} 3$ & 01 & $\mathrm{Fz}$ & $\mathrm{Pz}$ & $\mathrm{POz}$ & $\mathrm{Oz}$ & $\mathrm{O} 2$ & $\mathrm{PO} 4$ & PO8 & P6 & C6 & T8 & F6 \\
\hline \multicolumn{19}{|c|}{ Lower alpha } \\
\hline White & & - & & & & + & & & + & + & & & & & & _ & - & \\
\hline Black & + & + & & & & & & + & & 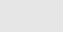 & & & & - & - & + & + & \\
\hline Gray & & - & - & & - & & & & & + & & & & & & - & - & \\
\hline Red & & - & - & - & - & & - & & & & - & - & - & - & - & & & \\
\hline Green & - & & & & & & & & & & & - & & - & - & - & - & - \\
\hline Blue & & + & + & - & - & - & - & - & - & - & - & & - & - & - & - & - & \\
\hline Yellow & - & - & - & & & & & & & + & + & & & - & - & & - & - \\
\hline Purple & + & & & - & - & & - & & & & - & - & & - & -- & - & - & \\
\hline \multicolumn{19}{|c|}{ Upper alpha } \\
\hline White & & + & + & + & & & & & & & & & + & + & + & & & + \\
\hline Black & & & & & & & & & - & - & - & - & - & - & - & & + & \\
\hline Gray & + & & & & & & & - & & & & & & & & & & \\
\hline Red & + & + & + & & & & & & & & & & & - & & + & + & \\
\hline Green & & & - & - & - & - & - & & - & - & - & - & - & - & - & & & \\
\hline Blue & - & - & - & - & - & - & - & - & - & - & - & - & - & - & - & & & - \\
\hline Yellow & - & - & - & 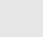 & - & 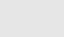 & - & - & - & - & - & - & & - & & & & - \\
\hline Purple & & & & - & - & - & & & & & & & & & & & & \\
\hline \multicolumn{19}{|l|}{ Gamma } \\
\hline White & & & & & & & & & & + & + & + & + & + & + & + & & \\
\hline Black & - & - & & + & + & + & + & - & + & + & + & + & & + & & & & 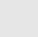 \\
\hline Gray & - & & & & & & + & & & + & + & + & + & + & & & & - \\
\hline Red & & & - & & & & & & & & & & & + & & & & - \\
\hline Green & - & & - & & & & & & & & + & & & & & & - & \\
\hline Blue & & & & & + & & + & & & + & + & + & + & + & + & & & \\
\hline Yellow & - & & & & + & & + & & + & + & + & + & + & + & + & & + & - \\
\hline Purple & - & & + & + & + & + & + & & & + & + & + & + & & + & & & - \\
\hline
\end{tabular}

Table 4

Electrodes showing significant increases (+) and decreases (-) of corrected EEG power for the different frequency bands and colors in participant KO

\begin{tabular}{|c|c|c|c|c|c|c|c|c|c|c|c|c|c|c|c|c|c|c|}
\hline & F5 & $\mathrm{T} 7$ & C5 & P5 & PO7 & PO3 & 01 & $\mathrm{Fz}$ & $\mathrm{Pz}$ & $\mathrm{POz}$ & $\mathrm{Oz}$ & $\mathrm{O} 2$ & PO4 & PO8 & P6 & C6 & T8 & F6 \\
\hline \multicolumn{19}{|c|}{ Lower alpha } \\
\hline White & - & & & & - & & & - & - & & & & & & & & & \\
\hline Gray & - & -- & - & & 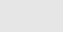 & & & - & - & - & - & - & - & - & - & - & - & - \\
\hline Red & - & - & - & - & - & - & - & . & - & - & -- & - & - & - & - & - & - & - \\
\hline Green & & & & + & + & + & + & + & & + & + & & & & & & + & + \\
\hline Blue & & - & & & - & - & - & & - & - & - & - & - & - & - & - & - & - \\
\hline Yellow & - & & & & & & & - & - & & & & & & & - & -- & - \\
\hline Purple & & & & & & & & & & & & + & & & & & - & \\
\hline \multicolumn{19}{|c|}{ Upper alpha } \\
\hline White & & + & + & & & & & & & & & & & & & - & - & - \\
\hline Gray & & & & & & & & & & & & & & & & & & \\
\hline Red & & & & + & + & + & + & & - & & & & & & & & & 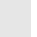 \\
\hline Green & & & & & & & & - & & & & & & & & + & & + \\
\hline Blue & + & & + & + & + & + & + & + & + & + & + & + & + & + & + & & & \\
\hline Yellow & & & & & & & & & - & - & & & & & & - & & \\
\hline Purple & + & + & & & & & & & & - & - & - & - & - & & & + & + \\
\hline \multicolumn{19}{|l|}{ Gamma } \\
\hline White & & - & - & & & & & & + & + & & & & + & & - & & \\
\hline Gray & - & & + & & + & & & & + & & + & + & & + & + & & + & \\
\hline Red & & & & & + & & & & & & & & & & & & + & \\
\hline Green & & - & & & - & & & & - & & + & & & & & & & \\
\hline Blue & & & + & & 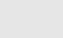 & + & + & & + & + & + & + & + & + & + & + & + & + \\
\hline Yellow & & + & + & & + & & & & + & & & & + & + & + & & + & \\
\hline Purple & & & & & + & & + & & & & + & & & - & & & & \\
\hline
\end{tabular}

KO did not report any occurrences of the color black during the experiment.

\section{Discussion}

Previous studies have indicated that while some hallucinatory colors are clearly associated with the experience of hallucinatory form, other colors do no co-occur with forms. Instead, they appear to represent an ambient change in color or illu- 


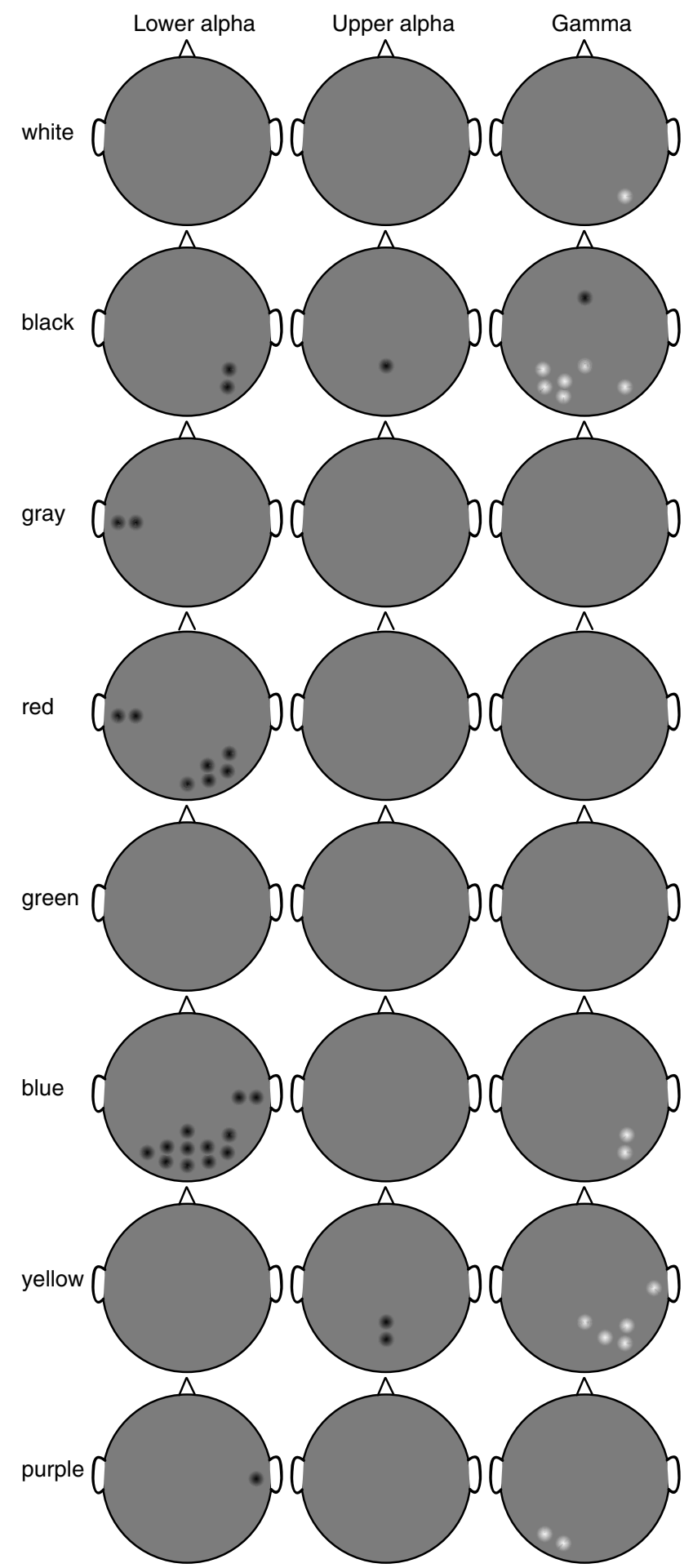

Fig. 2. Changes of corrected EEG power over time for the different EEG bandwidths and the different colors. The effects displayed were found to be significant in all three participants. The sign of the significant regression slopes is indicated by gray-scale: white represents electrodes at which a significant increase in EEG power over time was found, black represents a significant decrease of EEG power over time. A key of all electrode positions is given in Fig. 4.

mination (Becker \& Elliott, 2006; Fig. 7). These colors are yellow and blue alongside white and gray. Interestingly, in this experiment experience of these colors is, in each case, accompanied by an increase in gamma frequency power under right parieto-occipital (PO8), and, to a lesser extent, parietal (P6) electrodes. The fact that this parieto-occipital increase in gamma 


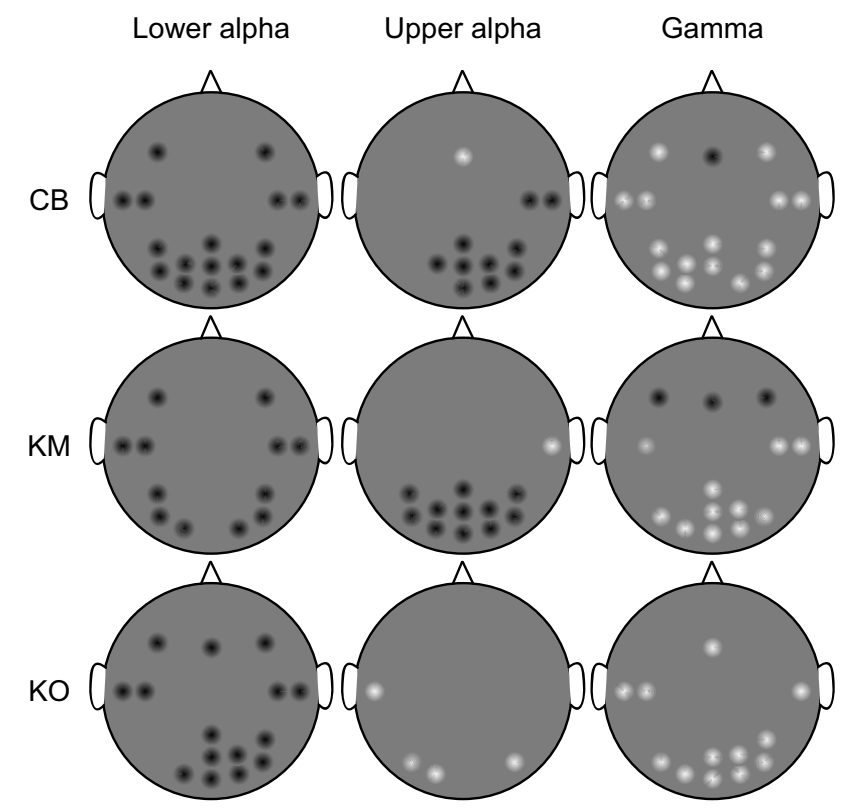

Fig. 3. Changes of the corrected EEG power over time for the different EEG bandwidths for the individual participants with the data being collapsed over all target colors. For information about the gray-scale coding see caption of Fig. 2. A key of all electrode positions is given in Fig. 4.

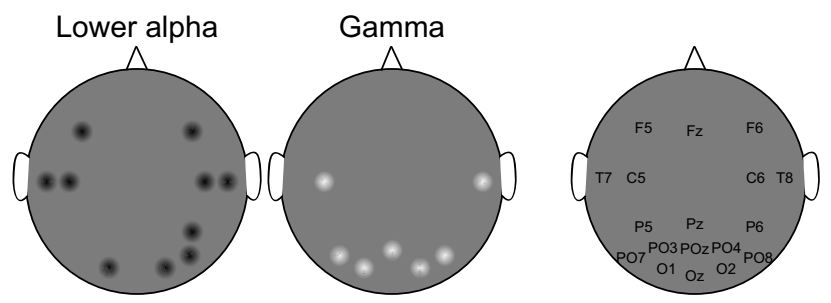

Fig. 4. Representation of changes of EEG power over time for the lower alpha and gamma EEG bandwidths found to be significant in all three participants. The right picture shows all electrode positions included in the analysis. For information about the gray-scale coding see caption of Fig. 2.

power is also observed for the experience of the color black might be indicative of the coding of ambient or scene luminance or contrast.

With respects to the experience of hallucinatory colors in general, changes in EEG power reveal a consistent picture across participants that agrees very well with the functional role ascribed to the different frequency bands in the literature. In particular, decreases in alpha and increases in gamma power under occipital and parieto-occipital electrodes seem a likely correlate of the formation of the hallucinatory, but nonetheless, clearly 'visual' representations induced by the flicker. The loci of these activity changes are also well in accordance with brain activation to colored stimuli measured using PET (Chao \& Martin, 1999) and fMRI (McKeefry \& Zeki, 1997).

As detailed in the introduction, specific changes of alpha activity have been reported during the perception of multistable figures (see for example, Müller et al., 2005 and Strüber \& Herrmann, 2002). Strüber and Herrmann (2002) suggest that alpha activity during the perception of one interpretation of a multistable figure constantly decreases until it reaches a critical threshold following which a perceptual switch occurs and a second interpretation of the figure is perceived. In the brain, this might be achieved by means of a decrease in alpha band synchronization, which would allow neurons to synchronize at other frequencies (such as those in the gamma band), responsible for the coding and representation of a new percept or event. The idea that low alpha activation might provide an endogenous and necessary condition for the conscious experience of a stimulus is also emphasized in the studies of Ergenoglu et al. (2004) and Hanslmayr et al. (2005). These studies show that a stimulus presented at threshold is more likely to be perceived when the alpha power is relatively low directly preceding stimulus presentation, while alpha desynchronization was especially pronounced in parietal and occipital cortices (Ergenoglu et al., 2004).

In our study, participants reported seeing a number of other colors preceding or co-occurring with the experience of the target color in the majority (90.5\%) of all trials. A multidimensional scaling of co-occurrent hallucinatory experiences in Becker and Elliott (2006) gave indications for inhibitory relations between certain colors (for example between blue and pur- 
ple; see Becker \& Elliott for a full description of the pattern of inter-relationships between colors), implying the impossibility of these colors to be experienced at the same time. In this respect, the experience of hallucinatory color may be similar to the perception of multistable figures (Müller et al., 2005; Strüber \& Herrmann, 2002), where one interpretation of the figure is deactivated and replaced by another interpretation. The decrease of alpha power preceding the report of hallucinatory color may reflect such a transition.

The topographic distribution of increased gamma power immediately preceding experience of hallucinatory color corresponds to the patterns of EEG activity observed during perception of multistable figures, e.g. Başar-Eroglu, Strüber, Kruse, Başar, and Stadler (1996a) who reported enhanced gamma band activation at frontal, parietal, and occipital areas preceding the switch in interpretation of a multistable percept. The combination of decreased alpha and increased gamma activity may thus reflect the necessary brain state for the experience of hallucinatory color with the corollary that this combination of brain states is a general, but contents-wise an almost entirely endogenous mechanism for color phenomenology. A more precise description of the mechanisms concerned remains speculative, particularly as concerns the tendency for hallucinatory experiences to change in color. We might speculate that a form of alpha gating in which alpha activity may be induced in the absence of specific perceptual stimuli, and thus in a similar fashion to that accompanying states of mental relaxation (Klimesch, 1999). One direct consequence would be the synchronization of cortical networks at alpha frequencies, which we argue to inhibit the formation of novel representations. Nonetheless, the coherence of alpha activity would very likely be susceptible to fluctuations due to the direct and indirect responses of neurons to the regular temporal patterning of the flickering ganzfeld. Alpha activity might decrease in coherence until it reaches some coherence threshold at which it is no longer sufficiently powerful to inhibit other neural networks. With a decrease in the inhibitory power brought about by alpha synchronization, concurrent color-specific activity at other frequencies, perhaps generated as early as the retina and perhaps represented by networks of synchronized gamma activity, would gain access to cortical processing mechanisms and emerge into the ongoing phenomenology experienced in the ganzfeld. Thus, a form of 'alpha gating', based upon relative patterns of synchronization and desynchronization across time and different neural networks might be responsible for the changes in color experienced when viewing the flickering ganzfeld. Importantly, the highly dynamic nature of the hallucinatory experiences induced by flicker stimulation requires a model of these conscious experiences that does not only allow a stationary description of the relation between the conscious phenomenal content and its neural representation, but aims to describe the relation between the two in its dynamic form encompassing all potential manifestations of the system. Fell (2004) outlined a promising approach to identify the neural correlates of conscious experience by equating the phenomenal state space of experiences with a neural state space of brain processing. While the perception of hallucinatory colors might be represented in a one-dimensional phenomenal state space, at least a two-dimensional representation of hallucinatory color seems more appropriate based on our data: This is because the relation of a hallucinatory color to a distinct spatial content can be expressed along one dimension (e.g. ambient color vs. shape-related color), with the second (and possibly further) dimension representing the actual color category produced by physiological color mechanisms. At least a three-dimensional neural state space would be required to successfully correlate the phenomenal with the neural state space. While the topographical distribution of electrophysiological brain responses is a vital component of this neural state space, more importantly, brain activity in at least two temporal domains, the alpha and gamma frequency band, is necessary to describe changes in phenomenal content that occur during an epoch of flicker. Further electrophysiological studies investigating the temporal succession of hallucinatory color need to be conducted in order to clarify the exact relation between a change of state in phenomenal state space (i.e. a switch in the perception of hallucinatory color) and a change of state in neural state space (e.g. changes in the topographical distribution of brain activity).

In summary, we show that EEG power changes preceding the experience of flicker-induced hallucinatory color are well in accordance with electrophysiological patterns of activity described in the literature on conscious perceptual processing. The perception of hallucinatory color is preceded by an inter-individually consistent decrease in power in the lower alpha frequency band alongside an increase in gamma frequency power. Decreasing alpha activity may reflect processes related to the generation of a new percept or a switch between different percepts. Increasing gamma band activation may similarly correlate with a perceptual switch; it may also indicate the formation of a coherent percept preceding the response. While the perception of hallucinatory color appears to be an entirely private experience of the observer, not accessible directly by the experimenter, the measurement of electrophysiological markers indicates the existence of a common objective set of brain processes underlying the highly subjective phenomenal content of consciousness.

\section{Acknowledgments}

This research was conducted at the LMU in Munich and was supported by German Research Foundation (DFG) project Grant EL 248/2 to M.A.E. and H.J.M.

\section{References}

Başar, E., Başar-Eroglu, C., Karakaş, S., \& Schürmann, M. (1999). Are cognitive processes manifested in event-related gamma, alpha, theta and delta oscillations in the EEG? Neuroscience Letters, 259(3), 165-168.

Başar-Eroglu, C., Strüber, D., Kruse, P., Başar, E., \& Stadler, M. (1996a). Frontal gamma-band enhancement during multistable visual perception. International Journal of Psychophysiology, 24(1-2), 113-125. 
Başar-Eroglu, C., Strüber, D., Schürmann, M., Stadler, M., \& Başar, E. (1996b). Gamma-band responses in the brain: A short review of psychophysiological correlates and functional significance. International Journal of Psychophysiology, 24(1-2), 101-112.

Becker, C., \& Elliott, M. A. (2006). Flicker induced color and form: Interdependencies and relation to stimulation frequency and phase. Consciousness \& Cognition, 15(1), 175-196.

Billock, V. A., \& Tsou, B. H. (2007). Neural interactions between flicker-induced self-organized visual hallucinations and physical stimuli. Proceedings of the National Academy of Sciences United States of America, 104(20), 8490-8495.

Chao, L. L., \& Martin, A. (1999). Cortical regions associated with perceiving, naming, and knowing about colors. Journal of Cognitive Neuroscience, 11(1), $25-35$.

Courtney, S. M., \& Buchsbaum, G. (1991). Temporal differences between color pathways within the retina as a possible origin of subjective colors. Vision Research, 31(9), 1541-1548.

Engel, A., Fries, P., \& Singer, W. (2001). Dynamic predictions: Oscillations and synchrony in top-down processing. Nature Reviews Neuroscience, 2(10), $704-716$.

Ergenoglu, T., Demiralp, T., Bayraktaroglu, Z., Ergen, M., Beydagi, H., \& Uresin, Y. (2004). Alpha rhythm of the EEG modulates visual detection performance in humans. Cognitive Brain Research, 20(3), 376-383.

Fell, J. (2004). Identifying neural correlates of consciousness: The state space approach. Consciousness and Cognition, 13(4), 709-729.

Grunfeld, E. D., \& Spitzer, H. (1995). Spatio-temporal model for subjective colors based on color coded ganglion cells. Vision Research, 35(2), $275-283$.

Hanslmayr, S., Klimesch, W., Sauseng, P., Gruber, W., Doppelmayr, M., Freunberger, R., et al (2005). Visual discrimination performance is related to decreased alpha amplitude but increased phase locking. Neuroscience Letters, 375(1), 64-68.

Herrmann, C. S., \& Mecklinger, A. (2001). Gamma activity in human EEG is related to high-speed memory comparisons during object selective attention. Visual Cognition, 8(3-4), 593-608.

Isoglu-Alkaç, U., Basar-Eroglu, C., Ademoglu, A., Demiralp, T., Miener, M., \& Stadler, M. (2000). Alpha activity decreases during the perception of Necker cube reversals: An application of wavelet transform. Biological Cybernetics, 82(4), 313-320.

Klimesch, W. (1997). EEG-alpha rhythms and memory processes. International Journal of Psychophysiology, 26(1-3), 319-340.

Klimesch, W. (1999). EEG alpha and theta oscillations reflect cognitive and memory performance: A review and analysis. Brain Research Reviews, 29(2-3), 169-295.

McKeefry, D. J., \& Zeki, S. (1997). The position and topography of the human colour centre as revealed by functional magnetic resonance imaging. Brain, 120(12), 2229-2242.

Mecklinger, A., Kramer, A., \& Strayer, D. (1992). Event-related potentials and EEG components in a semantic memory search task. Psychophysiology, 29(1), 104-119.

Müller, T. J., Federspiel, A., Fallgatter, A. J., \& Strik, W. K. (1999). EEG signs of vigilance fluctuations preceding perceptual flips in multistable illusionary motion. NeuroReport, 10(16), 3423-3427.

Müller, T. J., Federspiel, A., Horn, H., Lövblad, K., Lehmann, C., Dierks, T., et al (2005). The neurophysiological time pattern of illusionary visual perceptual transitions: A simultaneous EEG and fMRI study. International Journal of Psychophysiology, 55(3), $299-312$.

Niedermeyer, E. (1999). The normal EEG of the waking adult. In E. Niedermeyer \& F. H. Lopes da Silva (Eds.), Electroencephalography: Basic principles clinical applications and related fields (4th ed.). Philadelphia: Lippincott Williams \& Wilkins.

Shevelev, I. A., Kamenkovich, V. M., Bark, E. D., Verkhlutov, V. M., Sharaev, G. A., \& Mikhailova, E. S. (2000). Visual illusions and traveling alpha waves produced by flicker at alpha frequency. International Journal of Psychophysiology, 39(1), 9-20.

Shevelev, I. A., Kamenkovich, V. M., \& Sharaev, G. A. (1996). Visual illusions and alpha-rhythm EEG. Zhurnal Vysshei Nervnoi Deiatelnosti Im. I.P. Pavlova, 46(1), 34-39.

Strüber, D., \& Herrmann, C. S. (2002). MEG alpha activity decrease reflects destabilization of multistable percepts. Cognitive Brain Research, 14(3), 370-382.

Tallon, C., Bertrand, O., Bouchet, P., \& Pernier, J. (1995). Gamma-range activity evoked by coherent visual stimuli in humans. European Journal of Neuroscience, 7(6), 1285-1291.

Tallon-Baudry, C., \& Bertrand, O. (1999). Oscillatory gamma activity in humans and its role in object representation. Trends in Cognitive Sciences, 3(4), $151-162$.

Tiitinen, H., Sinkkonen, J., Reinikainen, K., Alho, K., Lavikainen, J., \& Näätänen, R. (1993). Selective attention enhances the auditory 40 Hz transient response in humans. Nature, 364, 59-60.

von Stein, A., Chiang, C., \& König, P. (2000). Top-down processing mediated by interareal synchronization. Proceedings of the National Academy of Sciences, 97(26), 14748-14753.

von Stein, A., \& Sarnthein, J. (2000). Different frequencies for different scales of cortical integration: From local gamma to long range alpha / theta synchronization. International Journal of Psychophysiology, 38(3), 301-313. 